\title{
Investigation of some pharmacological effects of Caffeine and Taurine in food supplements
}

\author{
Dobrina D. Tsvetkova ${ }^{1}$, Radoslav C. Klisurov ${ }^{2}$, Stefka A. Pankova ${ }^{1}$, Branimir A. Zlatkov ${ }^{1}$ \\ ${ }^{1}$ Department of Pharmaceutical Chemistry, Faculty of Pharmacy, Medical University - Sofia, Sofia, Bulgaria \\ ${ }^{2}$ Department of Pharmacology and Toxicology, Faculty of Medicine, Medical University - Sofia, Sofia, Bulgaria
}

\section{Email address:}

dobrinka30@abv.bg (D. D. Tsvetkova)

\section{To cite this article:}

Dobrina D. Tsvetkova, Radoslav C. Klisurov, Stefka A. Pankova, Branimir A. Zlatkov. Investigation of Some Pharmacological Effects of Caffeine and Taurine in Food Supplements. International Journal of Nutrition and Food Sciences. Special Issue: Taurine and Caffeine Supplementation in Energy Food Drinks: Uses, Side Effects and Quality Control. Vol. 4, No. 1-1, 2015, pp. 18-23.

doi: $10.11648 /$ j.ijnfs.s.2015040101.14

\begin{abstract}
In modern times in all age groups energy supplements containing different amounts of Caffeine and Taurine are applied. Caffeine is purine alkaloid, which stimulates central nervous system action, enhances the strength and frequency of the cardiac contractions and increases the excretion of urine. Taurine is a sulfur containing amino acid, which possesses many fundamental biological roles including: effect on synaptic transmission in the central nervous system, cardiotropic action, antioxidant and anticonvulsant activity, improvement of energy processes, stimulation of reparative processes in tissues, protection of eyes cataract, decrease of cholesterol and stimulation of immune system. The combination of Caffeine and Taurine provide benefit due to obtaining synergism of pharmacological effects in increasing of physical activity, stimulation of brain action, cognition, memory and attention. In connection with the significant enlarging of the consumption of energy drinks, especially by children and young people in recent years the requirements for regulation and control of the labeling of these products in many countries are enlarged. In many food additives Caffeine and Taurine are added in not labeled high concentrations, which can provoke and increase their side effects. High consumption of Caffeine enhances its adverse effects on body: anxiety, headache, insomnia, nervousness, respiratory disorders, tachycardia, tremor, dehydration. In children the adverse reactions of Caffeine in much lower doses than adults are occurred. In high concentrations Taurine has adverse effects on brain activity and can induce psoriasis. The result of combination of Caffeine and Taurine is associated with increased diuretic effect and loss of water and salts from the body, especially in children and young people. Because of these facts the quality and quantity control of included compounds in food supplements is important for their health safety.
\end{abstract}

Keywords: Caffeine, Taurine, Food Supplements, Pharmacological Effects

\section{Introduction}

\subsection{Energy Drinks as Dietary Supplements}

Dietary supplements are defined as "any product added to the total diet that contains at least one of the following ingredients: vitamin, mineral, herb or botanical, amino acid, metabolite, extract or combination of this ingredients" [1].

The consumption of energy additives from young people and athletes usually is enlarged. Food supplements contain different combinations and amounts of Caffeine, Taurine, vitamins from B group, plant extracts (guarana, mate, acacia, ginseng, ginkgo biloba), carnitine, creatine, glucuronolactone, inositol, carnitine and creatine. This compounds stimulate brain activity, memory and attention [1].

In recent years the requirements for regulation and control of the labeling of energy additives in many European countries, USA and Canada are increased. In many European countries the recommendations for limiting of the intake of energy products by children and pregnant women are approved. From Union of European Soft Drinks Associations many documents that include specific recommendations to manufacturers for the labeling of energy drinks has developed [2].

According to the European Authority for Food Safety the acute health and fatal problems occurred due to the high consumption of energy supplements alone or with alcohol or drugs substances or in combination with significantly exercise. Because of the increased consumption of energy additives 
from young athletes and active individuals and due to the variety of physiological and psychological effects of this products, in recent years different ingredients of energy drinks are the subject of research and evaluations [1]. The insufficiently explored interactions between the individual components of energy supplements require further investigation of their pharmacological effects. The quality and quantity control of included compounds in food additives is important for their health safety [2].

\section{Caffeine and Taurine.}

\subsection{Pharmacological Activity of Caffeine}

Caffeine (1,3,7-trimethylxanthine) (Fig. 1.) is purine alkaloid, which is one of the most consumed stimulant. Its natural sources are coffee tree (Coffea arabica), green tea (Camellia sinensis), Cola acuminata, guarana (Paulina cupana), cocoa and yerba maté (Ilex paraguariensis). By acting as a non selective $\mathrm{A} 1$ and $\mathrm{A} 2$ adenosine receptor antagonist it inhibits the adenosine suppressive activity. Actions of this alkaloid are associated with blocking the phosphodiesterase enzyme, which causes the accumulation of cyclic adenosinmonophosphate in the tissues [3].

Caffeine possesses different physiological effects: stimulates central nervous system action, enhances the mental activity and the physical performance, improves the cognition [4] and reaction time, exerts a positive effect on conditional reflexes, improves dopaminergic and cholinergic neurotransmission [3].<smiles>Cn1c(=O)c2c(ncn2C)n(C)c1=O</smiles>

Fig. 1. Chemical structure of Caffeine.

It increases blood pressure, heart rate [5] and the frequency and strength of heart contractions [6] and possesses analeptic action of the vasomotor and respiratory center in the brain [3].

Caffeine exerts diuretic activity by the following mechanisms:

1) decreasing of resorbtion of sodium and chlorine ions in the proximal kidney channels

2) improving the kidney hemodinamic and enhancing of the glomerulus filtration

3 ) increasing the plasma volume of the blood by lowering the hydrophilicity of the tissues

4) inhibition of the secretion of the antidiuretic hormone by stimulation of volumetric receptors [3].

Oxidative stress is imbalance between the overproduction of reactive oxygen species from endogenous or exogenous sources and the decrease in antioxidant defense mechanisms.
Oxidative stress is implicated in the pathogenesis of aging and different human diseases: neurodegenerative diseases including Alzheimer, Parkinson, Huntington, amyotrophic lateral sclerosis (Lou Gehrig's disease), Down's syndrome, atherosclerosis, vascular disease, cancer, diabetes mellitus type 1 and type 2, age-related macular degeneration, psoriatic arthritis [7].

Antioxidants protect cells of the body from damage that results from certain chemical reactions involving oxidation [7].

Antioxidant role of Caffeine is associated with scavenging the hydroxyl radical $(\mathrm{OH} \cdot)$ [8]. Its major metabolites 1-methylxanthine and methyluric acid are highly effective antioxidants [9, 10] (Fig. 2.).

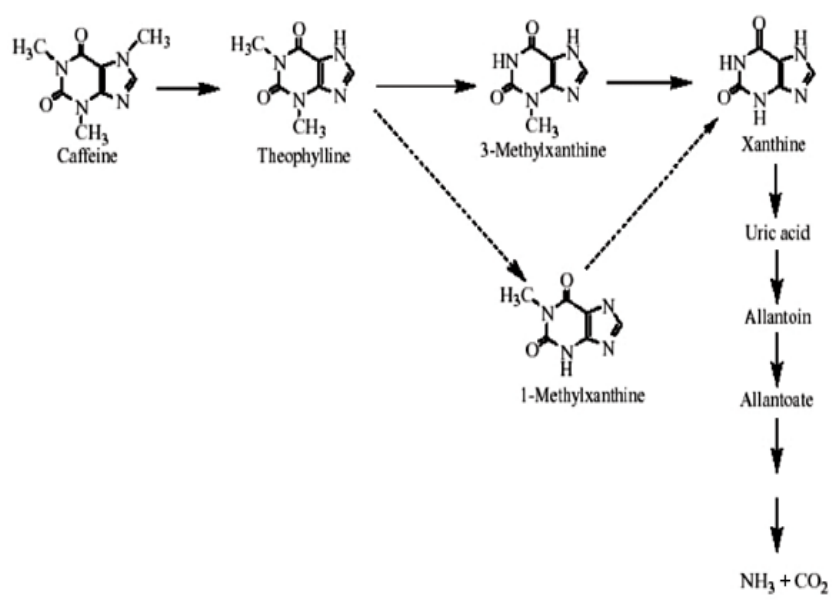

Fig. 2. Metabolism of Caffeine [10].

Caffeine prevents age-associated decline in cognitive function after its chronic application, through the protection of the antioxidant system in vivo [11].

Consuming of an energy drink with Caffeine $60 \mathrm{~min}$. before exercises improves mental focus, performance, concentration, reaction time, incites skeletal muscle metabolism and therefore it has been widely used in athletic competitions due to its ergogenic effects [12].

\subsection{Symptoms of Caffeine Overdose}

The pharmacokinetic properties of Caffeine are dose dependent, which contributes to toxic effects associated with many its overdoses in dietary supplements. Excessive amounts of Caffeine can adversely affect the body through significant toxic effects:

cetral: anxiety, delirium, headache, insomnia, nervousness systemic: dehydratation, hyperglycemia, fever heart: arrhythmia [13], severe hypertension, tachycardia respiratory disorders: troubled and rapid breathing; gastric tract: abdominal pain, diarrhea, nausea, vomiting, erosive changes in the lining of the gastrointestinal tract urinary: increased and frequent urination leading to dehydration due to loss of water and salts from the body muscular: tremor eyes: blurred vision mouth: dryness 
skin: flushing, increased sensitivity to touch or pain [3].

After repeated administration alkaloid leads to a weak dependency and at high doses causes exhaustion of neurons. In elderly its effect is accompanied with frequent awakenings. The full inhibition of the brain phosphodiesterase leads to convulsive effect at toxic concentrations of Caffeine [3].

Childrens are more vulnerable, because of the occurrence of adverse effects of Caffeine in much lower doses than adults. The combined intake of energy drinks with alcohol or narcotic drugs posses a significant health risk, especially in young people, as the effect of energy drinks masks the toxic effects of alcohol and narcotic drugs [3].

\subsection{Pharmacological Effects of Taurine}

Taurine (2-aminoethanesulfonic acid) (Fig. 3.) is a sulfur containing conditional amino acid, which is synthesized in the body as a result of metabolism of cysteine. This amino acid crosses the blood-brain barrier and is distributed in brain, heart, skeletal muscles, intestine, bile, liver, kidney, retina, leukocyts, platelets and is $0.1 \%$ of total human body weight [14].

In prematurely born infants the lack of the enzymes converting cystathionine to cysteine leads to its deficiency. Due to this reason supplementation with Taurine is necessary in infants. Taurine containing energy products are Red Bull, AMP Energy, Celsius, Full Throttle, 5-hour Energy, Monster Energy, NOS, Rockstar, Venom, [14].

Deficiency of Taurine leads to decreased oxygen consumption and adenosine triphosphate synthesis, resulting in the formation of superoxide and the creation of oxidative stress. A number of pathological conditions including abnormalities in central nervous system, epilepsy, myopathy, hypertension, cardiovascular dysfunctions like arrhythmia, cardiomyopathy and congestive heart failure, hypercholesterolemia, hepatic disorders, cystic fibrosis, retinal degeneration, cancer, immune impairment, retardation of growth and development are is associated with Taurine deficiency [15].

It is reported that food supplementation with Taurine prevents the progression of hypertension [16], coronary heart disease, endothelial dysfunction in young male, type 1 diabetics, hepatitis and hypercholesterolemia [15].

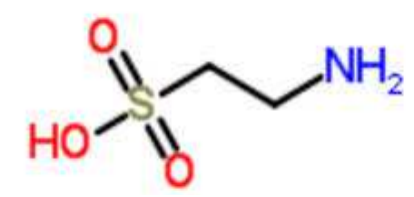

Fig. 3. Chemical structure of Taurine.

This amino acid exerts different pharmacological activity. Taurine possesses anxiolytic effect by activating the glycine receptor and exerts antidepressant, antiinflammatory, antiapoptotic [14] and antioxidant activity [17].

Taurine increases the development of nerve tissue and is important for synaptic transmission in central nervous system [18]. It protects against epileptic seizures and glutamate excitotoxicity [19]. In hypoxia in the brain very quickly is released glutamate, which leads to opening of the calcium channels and increasing of their concentration in neurons. Taurine plays neuroprotective role by interaction with GABA-receptors [15] and by regulation of cytoplasmic and mitochondrial calcium homeostasis due to inhibition of the glutamate-induced $\mathrm{Ca}^{2+}$ influx through L-, P/Q- and N-types of voltage gated calcium channels [20].

The reported facts that in patients with Alzheimer's disease are found decreased levels of Taurine and that this amino acid increases the levels of acetylcholine in the brain of animals, prove that Taurine can have beneficial effects in Alzheimer's disease and other neurological disorders due to blocking the neurotoxicity of $\beta$-amyloid by activation of GABA-receptors [20].

The normal function of dopaminergic neurons are controlled by NMDA-receptors. Their overaction leads to the accumulation of dopamine and neuronal death. Taurine reduces the release of dopamine acting as NMDA-receptor antagonist [15].

Taurine is essential for cardiovascular function and prevents from cardiovascular diseases [21], because improves the function of the left ventricle, exerts cardiotropic action, lowers blood pressure, stabilizes the heart rhythm, prevents platelet aggregation and regulates osmotic pressure [22]. It influences the heart, nervous system, blood vessels and the kidney in their contribution to arterial pressure regulation [23].

Antihypertensive effect of this amino acid is supported by the fact that in essential hypertensive patients after 6 weeks of treatment the systolic, diastolic and mean arterial blood pressure have been reduced [22].

Oral supplementation of Taurine induces antihypertensive effects in animal models of hypertension due to both central and peripheral effects. Specifically mediated by central nervous system effects of amino acid include modulation of baroreflex and regulation of the activity of hypothalamic neurons that are involved in secretion of vasopressin [23].

The vasorelaxation and hypotensive effects of Taurine are associated with its following peripherally mediated mechanisms:

1)reduction in plasma of levels of catecholamines adrenaline and noradrenaline

2)enhancing of natriuresis, urinary volume and kallikrein secretion

3)action as an osmoregulator on endothelial and vascular smooth muscle cells

4)increasing the expression of endothelial nitric oxide synthase, leading to enhancing the production of vasodilatator nitric oxide and stimulation of its release from the vascular endothelium;

5)minimizing the elevation of serum cytokine, endothelin, neuropeptide $\mathrm{Y}$ and thromboxane $\mathrm{B}_{2}$;

6)modulation of activity of the reninangiotensine-aldosterone system [24].

In endothelial cells antiapoptotic action of this amino acid is mediated via its antiinflammatory effect and ability for inhibition of reactive oxygen species. Taurine has also been shown to help people with congestive heart failure by 
increasing the force and effectiveness of heart-muscle contractions [24].

Supplementation with Taurine is possibly beneficial for the prevention of atherosclerosis, coronary heart disease and liver disease by decreasing of the secretion of apolipoprotein B100 which is structural component of VLDL and LDL [25].

This amino acid prevents the development of atherosclerosis and angiopathies by the following mechanisms:

1) lowering of serum lipids and lysophosphatidic acid, which is a lipid component in atherosclerotic plaques

2) reduction of the oxidation of LDL [24]

3 ) inhibition of platelet aggregation, which decreases the risk of formation of arterial thrombus [26].

Taurine reacts with phagocyte-derived hypochlorous acid resulting on formation of Tautochloramine. This compound inhibits synthesis of interleukine 6 and 8 and suppresses the physiological activation of matrix metalloproteases, which are secreted from intimal macrophages and foam cells. This leads to inhibition of proteolysis of collagen fibers and other protein components of the intercellular matrix and decreases the rupture of atherosclerotic plaque [24].

It is reported that Taurine suppresses the expression of myeloperoxidase - the enzyme which generates phagocyte-derived hypochlorous acid and by this mechanism lowers the risk of coronary disease and myocardial infarctions [27]. It works like a diuretic by keeping potassium and magnesium inside the cell and excessive sodium - out of the cell [24]. This amino acid decreases blood sugar and prevents diabetes associated microangiopathy and nephropathy [28].

The antioxidant effects of Taurine in biological systems is a result of its ability to scavenge reactive oxygen species, to stabilize biomembranes and to reduce the production of lipid peroxidation [14]. It decreases the oxidative stress also by inhibiting of the formation of advanced glycation endproducts [29].

It acts as an antioxidant and protects against toxicity of lead and cadmium and prevents many tissues of oxidant-induced toxic injury [30]. Supplementations with Taurine prevent oxidative stress induced by exercise [14].

Antineoplastic drugs lead to the development of secondary cancers in patients in extended remission. Due to its antioxidant properties Taurine acts against antineoplastic drugs-induced genotoxicity in somatic and germ tissues by reduction in DNA-fragmentation and decreasing of the frequency of chromosomal aberrations. These results prove that it may be of therapeutic potential in preventing the risk of secondary tumors in chemotherapy [31]. Taurine is chemopreventive agent against the colonic and hepatic cancers and have beneficial effects in acute hepatitis. Its derivates Tauromustine and Taurolidine exerts antineoplastic activity [20].

This amino acid is important for early embryonic development, immune system [32] and for function of skeletal muscles [33]. Taurine exerts positive effect by protecting eyes from dystrophic process in retina and from developing of diabetic, senile, radiation or traumatic cataract [14].
It is conjugated in the liver with chenodeoxycholic acid and cholic acid and form the bile salts sodium taurochenodeoxycholate and sodium taurocholate [34]. Taurine is involved in the formation of the surface-active substances, which promote the emulsification of the fatty acids in the diet [14].

This amino acid is in high concentrations in skeletal muscle, modulates contractile function and increases force generation by augmenting the accumulation and release of calcium from the sarcoplasmic reticulum [35].

Taurine helps to cell proliferation and viability and stabilization of the membranes. Physiologically it plays an important role in the abnormalities in the metabolism and stimulates of reparative processes in tissues. This amino acid improves energy processes, detoxifies some xenobiotics, regulates levels of calcium ions in blood, exerts hypolipidemic activity by its role of fatty tissues metabosm [14].

Taurine participates in the regulation of secretion of secretion of vasopressin and oxytocin. It has been shown that it exerts protective effect on human hair follicle grown in vitro [14].

Trimethyltaurine by binding to GABA-A receptors exerts neuroprotective properties. Tauropyrone protects cells from glutamate-induced cell death. Taurine chloramine and Taurine bromamine exhibit immunoregulatory activity [20].

\subsection{Symptoms of Taurine Overdose}

The increased plasma concentrations of growth hormone in some epileptic patients during administration of $50 \mathrm{mg} / \mathrm{kg}$ body mass per day Taurine, suggest a potential of this amino acid to stimulate the hypothalamus and to modify the neuroendocrine function, which can be a side effect in hight consumption of Taurine. There are found an indications that in dose $2 \mathrm{~g}$ per os daily Taurine can induce psoriasis[14].

The absorption of this amino acid from beverages is more rapid than from foods, which faster can provoke side effects in hight consumption. The combined administration of both Caffeine and Taurine is associated with increased diuretic effect and loss of water and salts from the body, especially in children and young people [14].

\subsection{Effects of Energy Supplements, Containing Combination of Caffeine and Taurine}

The consumption of energy drinks is exponentially increasing worldwide due to their stimulant effect on the central nervous system and body and the purpose of enhancing both cognitive and physical performances [36].

The introduction of Red Bull to the market in 1987 started a new beverage category: the "energy drink". Red Bull is the most consumed energy drink and in $250 \mathrm{ml}$ contains $80 \mathrm{mg}$ $(0.32 \mathrm{mg} / \mathrm{ml})$ Caffeine and $1000 \mathrm{mg}(4 \mathrm{mg} / \mathrm{ml})$ Taurine [36].

It is reported that Red Bull energy drink causes increased diastolic blood pressure, aerobic endurance and anaerobic performance. Taurine shows antihypertensive properties, lowering the systolic blood pressure [20]. 
The European Union's Scientific Committee on Food has published a report in March 2003 summarizing the investigation into potential interactions of the ingredients in energy drinks. At the cardiovascular level the conclusion is that Taurine might reduce the cardiovascular effects of Caffeine and can decrease Caffeine-mediated increase in systolic blood pressure [20,37].

The consumption of Caffeine and other stimulants have potentially beneficial effects on cognition, including improved attention and enhanced information processing and improve mental function [36]. It is demonstrated that energy drinks containing Caffeine and Taurine improve mood, cognition and memory [38] and increase mental performance, concentration and information processing [20].

Sports drinks are among the most widely used nutritional supplements. Combination of $80 \mathrm{mg}$ Caffeine and $1000 \mathrm{mg}$ Taurine posses performance-enhancing effects [39] in athletics. Supplements containing a combination of Caffeine and Taurine have been shown to enhance ventricular function by increasing stroke volume [35].

The duration of administration is important for effects of Caffeine and Taurine in energy drinks. This is confirmed by experimental results proving the that after two weeks an additive effects of Caffeine and Taurine occur and no additive effect is observed after only a week administration [40].

In patients the novel drug analgesic combination Tramadol $37.5 \mathrm{mg} /$ Paracetamol $325 \mathrm{mg} /$ Caffeine $30 \mathrm{mg} /$ Taurine 250 mg compared to Tramadol $37.5 \mathrm{mg} /$ Paracetamol $325 \mathrm{mg}$ exerts a superior analgesic effect and decreases adverse reactions like nausea, vomiting and dizziness [41].

\subsection{Ovedose of Combination of Caffeine and Taurine}

High consumption of energy drinks containing overdose of Caffeine and Taurine lead to health risks and toxicity [42], because of a variety of adverse health effects [36].

The combined administration of Caffeine and Taurine decreases heart rate. In comparison with intake of $80 \mathrm{mg}$ Caffeine the consumption of $80 \mathrm{mg}$ Caffeine and $1000 \mathrm{mg}$ of Taurine increases the blood pressure [43].

In consumption of the energy drink with Taurine during exercises it lead to cardiovascular effects in significant increase in contractility and a higher stroke volume. It is observed that the combination of exercises and the consumption of a Caffeine and Taurine-containing beverage leads to a heart attack [44].

Highly caffeinated beverage containing Taurine can lead to atrial fibrillation [45].

\section{Conclusion}

Caffeine is involved in a number of important physiological processes. Taurine participates in a great number of functions in the organism and maintains cellular homeostasis.

The synergism effect of these compounds is important due to improvement of mood, cognition and memory and increase mental performance.

There are many different types of caffeinated foods and beverages in which the content of Caffeine and Taurine is not labeled and in high consumption this can provoke side effects of two components.

Because of these facts the quality and quantity control of included compounds in food supplements is important for their health safety. This is the reason for increasing the requirements for their control in food additives.

\section{References}

[1] Dietary Supplement Health and Education Act (DSHEA), Public Law, Act 42 U.S.C. 287C-11, 1994, 103-417.

[2] K.A. Clauson, K.M. Shields, C.E McQueen, N. Persad, Safety issues associated with commercially available energy drinks, J. Am. Pharm. Assoc., 2008, 48(3), 55-63.

[3] B.B. Fredholm, Notes on the history of Caffeine use, Handb. Exp. Pharmacol., 2011, 200, 1-9.

[4] T.T. Brunyé, C.R. Mahoney, H.R. Lieberman, G.E. Giles, H.A. Taylor, Acute Caffeine consumption enhances the executive control of visual attention in habitual consumers, Brain and Cognition, 2010, 74, 186-192.

[5] R.J. Bloomer, I.C. Harvey, T.M. Farney, Z.W. Bell, R.E. Canale, Effects of 1,3-dimethylamyl-amine and Caffeine alone or in combination on heart rate and blood pressure in healthy man and women, Phys. Sportsmed., 2011, 39(3), 111-120.

[6] N.P. Riksen, P. Smits, G.A. Rongen, The cardiovascular effects of methylxanthines, Handb. Exp. Pharmacol., 2011, 200, 413-437.

[7] O. Firuzi, R. Miri, M. Tavakkoli, L. Saso, Antioxidant therapy: current status and future prospects, Curr. Med. Chem., 2011, 18(25), 3871-3888.

[8] R.P. Barcelos, M.A. Souza, G.P. Amaral, S.T. Stefanello, G. Bresciani, M.R. Fighera, F.A. Soares, N.V. Barbosa, Caffeine supplementation modulates oxidative stress markers in the liver of trained rats, Life Sci., 2014, 96(1-2), 40-45.

[9] F. Zeidán-Chuliá, D.P. Gelain, E.A Kolling, J.L. Rybarczyk-Filho, P. Ambrosi, S.R. Terra, A.S. Pires, J.B. da Rocha, G.A. Behr, J.C. Moreira, Major components of energy drinks (caffeine, taurine, and guarana) exert cytotoxic effects on human neuronal SH-SY5Y cells by decreasing reactive oxygen species production, Oxid. Med. Cell. Longev., 2013, 2013, 1-22.

[10] H. Ashihara, Metabolism of alkaloids in coffee plants, Braz. J. Plant Physiol., 2006, 18 (1), 1-8.

[11] R.V. Abreu, E.M. Silva-Oliveira, M.F. Moraes, G.S. Pereira, T. Moraes-Santos, Chronic coffee and Caffeine ingestion effects on the cognitive function and antioxidant system of rat brains, Pharmacol. Biochem. Behav., 2011, 99(4), 659-664.

[12] M.A. Tarnopolsky, Effect of Caffeine on the neuromuscular system-potential as an ergogenic aid, Appl. Physiol. Nutr. Metab., 2008, 33(6), 1284-1289.

[13] D.J. Pelchovitz, J.J. Goldberger, Caffeine and cardiac arrhythmias: a review of the evidence, Am. J. Med., 2011, 124(4), 284-289. 
[14] M. Bidri, P. Choay, Taurine: a particular amino acid with multiple functions, Ann. Pharm. Fr., 2003, 61(6), 385-391.

[15] Y. Yamori, T. Taguchi, A. Hamada, K. Kunimasa, H. Mori, M. Mori, Taurine in health and diseases: consistent evidence from experimental and epidemiological studies, J. Biomed. Sci., 2010, 17(Suppl 1), S6.

[16] M.M. Rahman, H.M. Park, S.J. Kim, H.K. Go, G.B. Kim, C.U. Hong, Y.U. Lee, S.Z. Kim, J.S. Kim, H.S. Kang, Taurine prevents hypertension and increases exercise capacity in rats with fructose-induced hypertension, Am. J. Hypertens., 2011, 24(5), 574-581.

[17] M. Sinha, P. Manna, P.C. Sil, Taurine protects the antioxidant defense system in the erythrocytes of cadmium treated mice, BMB Rep., 2008, 41(9), 657-663.

[18] J.Y. Wu, H. Prentice, Role of Taurine in the central nervous system, J. Biomed. Sci., 2010, 17, 1, S1.

[19] R. Leon, H. Wu, Y. Jin, J. Wei, C. Buddhala, H. Prentice, J.Y. $\mathrm{Wu}$, Protective function of Taurine in glutamate-induced apoptosis in cultured neurons, J. Neurosci. Res., 2009, 87(5), $1185-1194$.

[20] S.S. Oja, P. Saransaari, Pharmacology of Taurine, Proc. West. Pharmacol. Soc., 2007, 50, 8-15.

[21] A. Zulli, Taurine in cardiovascular disease, Curr. Opin. Clin. Nutr. Metab. Care, 2011, 14(1), 57-60.

[22] 29. A. El-Idrissi, E. Okeke, X. Yan, F. Sidime, L.S. Neuwirth, Taurine regulation of blood pressure and vasoactivity, Adv. Exp. Med. Biol., 2013, 775, 407-425.

[23] S. Roysommuti, J.M. Wyss, Taurine effects on arterial pressure control bioactive food as dietary interventions for cardiovascular disease. In: Bioactive food as dietary interventions for cardiovascular disease, 2013, Chapter 10, 183-195.

[24] W. Abebe, M.S. Mozaffari, Role of Taurine in the vasculature: an overview of experimental and human studies, Am. J. Cardiovasc. Dis., 2011, 1(3), 293-311.

[25] T.Yanagita, S.Y. Han, Y. Hu, K. Nagao, H. Kitajima, S. Murakami, Taurine reduces the secretion of apolipoprotein B100 and lipids in HepG2 cells, Lipids Health Dis., 2008, 7, 38.

[26] Y. Ijiri, H. Ikarugi, Y. Tamura, M. Ura, M. Morishita, A. Hamada, M. Mori, H. Mori, Y. Yamori, H. Ishii, J. Yamamoto, Antithrombotic effect of Taurine in healthy Japanese people may be related to an increased endogenous thrombolytic activity, Thromb. Res., 2013, 131(2), 158-161.

[27] M.F. McCarty, Supplementary Taurine may stabilize atheromatous plaque by antagonizing the activation of metalloproteinases by hypochlorous acid, Med. Hypotheses, 2004, 63(3), 414-418.

[28] J. Das, P.C. Sil, Taurine ameliorates alloxan-induced diabetic renal injury, oxidative stress-related signaling pathways and apoptosis in rats, Amino Acids, 2012, 43(4), 1509-1523.

[29] J.S. Huang, L.Y. Chuang, J.Y. Guh, Y.L.Yang, M.S. Hsu, Effect of Taurine on advanced glycation end products-induced hypertrophy in renal tubular epithelial cells, Toxicol. Appl. Pharmacol., 2008, 233(2), 220-226.
[30] C.J. Jong, J. Azuma, S. Schaffer, Mechanism underlying the antioxidant activity of taurine: prevention of mitochondrial oxidant production, Amino Acids 2012, 42(6), 2223-2232.

[31] S.S. Alam, N.A. Hafiz, A H. Abd-El-Rahim, Protective role of Taurine against genotoxic damage in mice treated with methotrexate and tamoxfine, Envir. Toxicol. Pharmacol., 2011, $31(1), 143-152$.

[32] J. Marcinkiewicz. Taurine bromamine (TauBr) - its role in immunity and new perspectives for clinical use, J. Biomed. Sci., 2010, 17, 1, S1-S3.

[33] L.A. Silva, P.C. Silveira, M.M. Ronsani, P.S. Souza, D. Scheffer, L.C. Vieira, M. Benetti, C.T. De, R.A. Pinho, Taurine supplementation decreases oxidative stress in skeletal muscle after eccentric exercise, Cell Biochem. Funct., 2011, 29(1), $43-49$.

[34] G.B. Draganov, I.P. Pencheva, K.A. Todorova, UV-spectrophotometry determination of taurine in energy drink mixtures, Int. J. Nutr. Food Sci., 2014, 3(2), 123-126.

[35] N. Gwacham, D.R. Wagner, Acute effects of a Caffeine-Taurine energy drink on repeated sprint performance of american college football players, Int. J. Sport Nutr. Exerc. Metab., 2012, 22(2), 109-116.

[36] J.P. Higgins, T.D. Tuttle, C.L. Higgins, Energy beverages: content and safety, Mayo Clin. Proceed., 2010, 85(11), 1033-1041.

[37] S.W. Schaffer, K. Shimada, C.J. Jong, T. Ito, J. Azuma, K. Takahashi, Effect of Taurine and potential interactions with Caffeine on cardiovascular function. Amino Acids, 2014, 46(5), 1147-1157.

[38] G.E. Giles, C.R. Mahoney, T.T. Brunyé, A.L. Gardony, H.A. Taylor, R.B. Kanarek, Differential cognitive effects of energy drink ingredients: Caffeine, Taurine, and glucose, Pharmacol. Biochem. Behav., 2012, 102(4), 569-577.

[39] A. Peacock, F.H. Martin, A. Carr, Energy drink ingredients. Contribution of Caffeine and Taurine to performance outcomes, Appetite, 2013, 64, 1-4.

[40] T.F. Imagawa, I. Hirano, K. Utsuki, M. Horie, A. Naka, K. Matsumoto, S. Imagawa, Caffeine and Taurine enhance endurance performance, Int. J. Sports Med., 2009, 30(7), 485-488.

[41] S.K. Madhusudhan, Novel analgesic combination of Tramadol, Paracetamol, Caffeine and Taurine in the management of moderate to moderately severe acute low back pain, J. Orthop., 2013, 10(3), 144-148.

[42] N. Gunja, J.A. Brown, Energy drinks: health risks and toxicity. Med. J. Aust., 2012, 196(1), 46-49.

[43] A.M., Franks, J.M. Schmidt, K.R. McCain, M. Fraer, Comparison of the effects of energy drink versus caffeine supplementation on indices of 24-hour ambulatory blood pressure, Ann. Pharmacother., 2012, 46(2), 192-199.

[44] A.J. Berger, K. Alford, Cardiac arrest in a young man following excess consumption of caffeinated "energy drinks", Med. J. Aust., 2009, 190(1), 41-43.

[45] J.R. DiRocco, A. During, P.J. Morelli, M. Heyden, T.A. Biancaniello, Atrial fibrillation in healthy adolescents after highly caffeinated beverage consumption: two case reports, J. Med. Case Rep., 2011, 5, 18. 